\title{
Characterization of the ultra-short echo time magnetic resonance (UTE MR) collagen signal associated with myocardial fibrosis
}

Adrienne G Siu ${ }^{1,2^{*}}$, Andrew Ramadeen ${ }^{3}$, Xudong Hư ${ }^{3}$, Lily Morikawa ${ }^{4}$, Li Zhang ${ }^{1,2}$, Justin Lau ${ }^{1,2}$, Garry Liu ${ }^{1,2}$, Mihaela Pop ${ }^{1,2}$, Kim A Connelly, $y^{5,3}$, Paul Dorian ${ }^{5,3}$, Graham A Wright ${ }^{1,2}$

From 18th Annual SCMR Scientific Sessions

Nice, France. 4-7 February 2015

\section{Background}

The homogeneous distribution of collagen in diffuse myocardial fibrosis renders the disease unsuitable for imaging using late gadolinium enhancement (LGE) [1]. More recently, the estimation of extracellular volume from $\mathrm{T}_{1}$ maps involving gadolinium agents has shown promise; however, these methods are not specific to collagen and are governed by gadolinium kinetics [2]. The diagnosis of diffuse myocardial fibrosis would benefit from an imaging method that can directly detect collagen. Notably, ultra-short echo time magnetic resonance (UTE MR) is a technique that can be used to detect short $\mathrm{T}_{2}{ }^{*}$ species, including collagen [3]. Our objective is to characterize the UTE signal of protons in the collagen molecule, including their $\mathrm{T}_{2}{ }^{*}$ and chemical shift. Direct isolation of a collagen signal could aid in the diagnosis of myocardial fibrosis, especially for diffuse distributions, and the assessment of disease extent.

\section{Methods}

Collagen solutions of concentrations ranging from $0 \% \mathrm{~m} / \mathrm{v}$ to $50 \% \mathrm{~m} / \mathrm{v}$ were prepared by dissolving hydrolyzed type I and III collagen powder in $0.125 \mathrm{mM} \mathrm{MnCl}_{2}$, where the signal decay of $\mathrm{MnCl}_{2}$ mimicked that of cardiac muscle. Each solution was scanned using a 3D UTE pulse sequence at $7 \mathrm{~T}$, acquiring TEs from $0.02 \mathrm{~ms}$ to $25 \mathrm{~ms}$, at a resolution of $0.781 \mathrm{~mm}$ isotropic. Upon fitting with a model of bi-exponential $\mathrm{T}_{2}{ }^{*}$ with oscillation, the UTE collagen signal fraction was determined and calibrated against the collagen concentration. The $\mathrm{T}_{2}{ }^{*}$ and resonance frequency (arising from the chemical shift) of collagen were assessed in

\footnotetext{
'Department of Medical Biophysics, University of Toronto, Toronto, ON, Canada

Full list of author information is available at the end of the article
}

collagen solutions. Validation of the collagen signal properties was also performed in formalin-fixed canine heart tissue, imaged with TEs from $0.02 \mathrm{~ms}$ to $25 \mathrm{~ms}$, at a resolution of $0.156 \mathrm{~mm}$ isotropic.

(a)

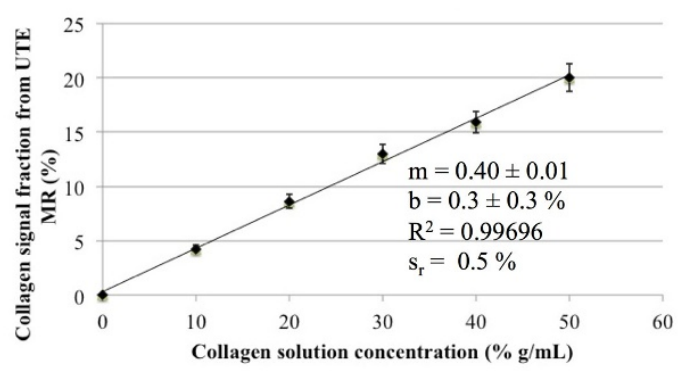

(b)

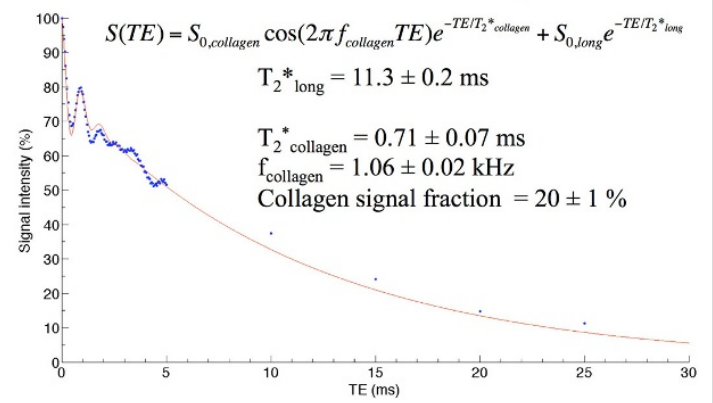

Figure 1 UTE results in collagen solutions. (a) Collagen solution calibration plot, demonstrating a linear relationship between the UTE collagen signal fraction and the collagen concentration. $m=$ slope, $\mathrm{b}=\mathrm{y}$-intercept, $\mathrm{R}^{2}=$ correlation coefficient, $\mathrm{s}_{\mathrm{r}}=$ standard deviation about the regression. (b) $T_{2}{ }^{*}$ decay of the $50 \%$ collagen solution, fitted using a bi-exponential $\mathrm{T}_{2}{ }^{*}$ model with oscillation. $\mathrm{T}_{2}{ }^{*}$ long denotes the long $\mathrm{T}_{2}^{*}$ of $\mathrm{MnCl}_{2}$ (mimicking cardiac muscle). Although not all long TEs were fitted, the focus was in the characterization of the short TEs $\leq$ $2 \mathrm{~ms}$, where the $\mathrm{T}_{2}{ }^{*}$ model is accurate. 


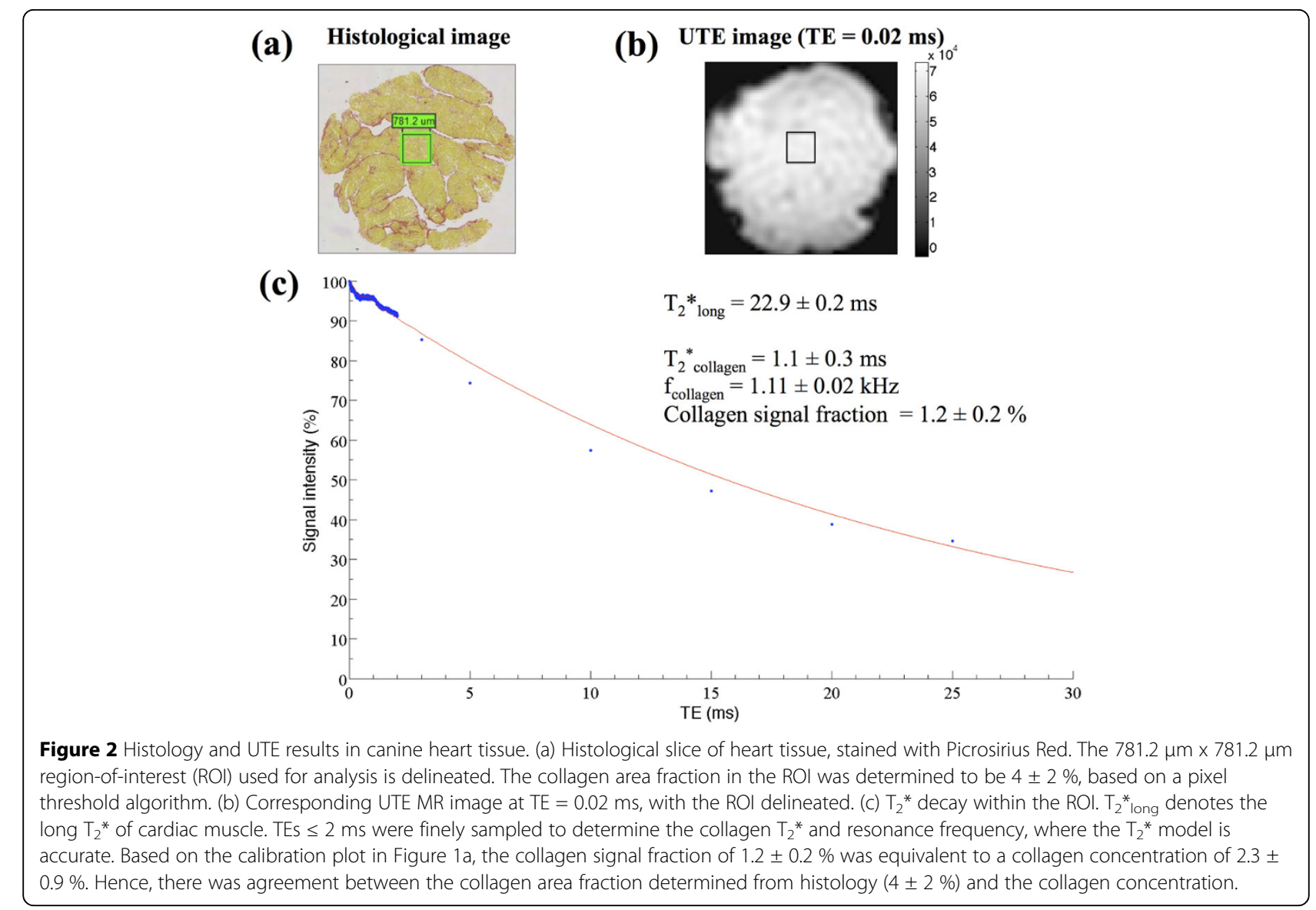

\section{Results}

For collagen concentrations of $10 \%$ to $50 \%$, the mean collagen $\mathrm{T}_{2}{ }^{*}$ was $0.75 \pm 0.05 \mathrm{~ms}$, and the mean collagen frequency was $1.061 \pm 0.004 \mathrm{kHz}$. A linear relationship (slope $=0.40 \pm 0.01, \mathrm{R}^{2}=0.99696$ ) was determined between the UTE collagen signal fraction associated with these characteristics and the measured collagen concentration (Figure 1). Similarly in canine heart tissue, a signal with $\mathrm{T}_{2}{ }^{*}$ of $1.1 \pm 0.3 \mathrm{~ms}$ and resonance frequency of $1.11 \pm 0.02 \mathrm{kHz}$ upfield of water was determined, consistent with collagen (Figure 2). The UTE collagen signal fraction of $1.2 \pm 0.2 \%$ in tissue corresponded to a collagen concentration of $2.3 \pm 0.9 \%$, which was within the uncertainty of the collagen area fraction determined from histology $(4 \pm 2 \%)$.

\section{Conclusions}

The results suggest that collagen associated with myocardial fibrosis can be endogenously detected and quantified using UTE MRI. This signal is specific to protons in collagen, characterized by a $\mathrm{T}_{2} *$ of $\sim 0.8 \mathrm{~ms}$ and $\mathrm{a}$ resonance frequency of $\sim 1.1 \mathrm{kHz}$ upfield of water at $7 \mathrm{~T}$. Such properties would be beneficial in the determination of collagen content due to disease.

\section{Funding}

Canadian Institutes of Health Research (CIHR).

\section{Authors' details}

'Department of Medical Biophysics, University of Toronto, Toronto, ON, Canada. ${ }^{2}$ Imaging Research, Sunnybrook Research Institute, Toronto, ON, Canada. ${ }^{3}$ Keenan Research Centre, Li Ka Shing Knowledge Institute, Toronto, ON, Canada. ${ }^{4}$ Centre for Modeling Human Disease, Toronto Centre for Phenogenomics, Toronto, ON, Canada. ${ }^{5}$ Division of Cardiology, St. Michael's Hospital, Toronto, ON, Canada.

Published: 3 February 2015

\section{References}

1. Sado, et al: Future Cardiol 2011.

2. Mewton, et al: J Am Coll Cardiol 2011.

3. De Jong, et al: J Moll Cell Cardiol 2011.

doi:10.1186/1532-429X-17-S1-Q7

Cite this article as: Siu et al:: Characterization of the ultra-short echo time magnetic resonance (UTE MR) collagen signal associated with myocardial fibrosis. Journal of Cardiovascular Magnetic Resonance 2015 17(Suppl 1):Q7. 